\title{
Structural Study of SrTi03 Doped with Mn Using X-Ray Diffraction
}

\author{
Zainab Ibrahim Booq, Sara Khalid Alghaith \\ Science College of King Saud University, Department of Physics and Astronomy \\ Turky Alaual, Riyadh, middle, Saudi Arabia, 11451 \\ Zbooq@ksu.edu.sa; S.al_ghaith@hotmail.com
}

\begin{abstract}
Monocrystalline $\mathrm{Mn}: \mathrm{SrTiO}_{3}$ was prepared from the compounds $\mathrm{MnO}_{2}, \mathrm{TiO}_{2}$, and $\mathrm{SrCO}_{3}$, which had equivalent weights of $2.52 \mathrm{~g}$. Mn was doped in this sample at $60 \mathrm{~mol} \%$. The sample was heated half hour, and annealed at $1223 \mathrm{~K}\left(950^{\circ} \mathrm{C}\right.$ ). The sample had an orientation of (100). The study was performed at room temperature $\left(T=300 \mathrm{~K}=27^{\circ} \mathrm{C}\right.$ ). The structure of this crystal was investigated using $X$-ray diffraction and found to be cubic $(P m \overline{3} m)$. The lattice parameter of the $\mathrm{Mn}: \mathrm{SrTiO}_{3}$ crystal was 3.269 A. The grain size of this crystal was magnified 5,000-100,000 times using scanning electron microscopy and found to be in range of $1.33 \mu \mathrm{m}-91.4 \mathrm{~nm}$.
\end{abstract}

Keywords: Spectrum, grain, crystal structure factor, atomic scattering factor, annealing

(C) Copyright 2017 Authors - This is an Open Access article published under the Creative Commons Attribution License terms (http://creativecommons.org/licenses/by/3.0). Unrestricted use, distribution, and reproduction in any medium are permitted, provided the original work is properly cited.

\section{Introduction}

is $\begin{array}{lll}\text { Strontium titanate oxide } & \mathrm{SrTiO}_{3} \text { or } \mathrm{STO} \text { ) crystal } \\ \text { oxide perovskite material }\end{array}$ $\left(\mathrm{ABO}_{3}\right.$, where $\mathrm{A}$ is the rare earth element, $\mathrm{B}$ is the titanate, and $\mathrm{O}_{3}$ is the oxide ) [1]. This group of materials has a wide range of applications, including uses in transistors, receivers, and non-vital memory devices [2]. It is a semiconductor material [3], but becomes a superconductor when doped with Mn or any other transition metal ion [4, 2, 5]. This characteristic is responsible for the development of numerous physical properties, including good conductivity and magnetic properties [4, 6].

Date Received: 2016-05-31

Date Accepted: 2016-11-22

Date Published: 2017-07-14
It is worth mentioning that the preparation methods for most $\mathrm{ABO}_{3}$ crystals are very similar. Several preparation techniques have been developed to produce $\mathrm{Mn}: \mathrm{SrTiO}_{3}$ nanoparticles, including a sol-gel method, organic precursor method [7], powder sample method [8], and solution method at high temperatures $[4,8,9,10]$.

In this study, a monocrystalline sample of $\mathrm{Mn}: \mathrm{SrTiO}_{3}$ was prepared using a solution method at a high temperature, where $\mathrm{Mn}$ was used to dope the $\mathrm{SrTiO}_{3}$ crystal $[4,8,9,10]$. Then, X-ray diffraction (XRD) was used to investigate the structure. This was the first study to investigate the structure of this crystal using a lowresolution XRD device with a maximum current intensity was $\mathrm{I}=1 \mathrm{~mA}$ and voltage $\mathrm{V}=25 \mathrm{kV}$. The grain size was determined using scanning electron microscopy (SEM). The crystal preparation was complicated because available literature is not sufficiently clear, and there are different methods for doping the crystal and annealing at high temperatures, which requires a special furnace. In addition, special skill is required to adapt the lowresolution XRD device.

\section{Material and Methods \\ 2.1. Preparation of $\mathrm{Mn}: \mathrm{SrTiO}_{3}$ Monocrystal}

The $\mathrm{Mn}: \mathrm{SrTiO}_{3}$ monocrystal was prepared using powders of $\mathrm{MnO}_{2}, \mathrm{TiO}_{2}$, and $\mathrm{SrCO}_{3}[4,8,9,10]$ with purities of $70 \%, 99 \%$, and $98 \%$, respectively. These compounds were added by equal weights of 2.52. g of each of the compounds were used [8] , so that $\mathrm{SrTiO}_{3}$ was heavily doped by $\mathrm{Mn}$ at $60 \mathrm{~mol} \%$. The powders were mixed under magnetic stirring for $1 \mathrm{~h}$ to obtain a homogenous mixture. Then, the mixture was soaked in 
de-ionized (DI) water for 20-25 min [11, 12]. A 3:1 by volume HCL: $\mathrm{HNO}_{3}$ acidic solution was added and mixed for $12 \min [13,12]$. The sample was annealed by being heated in a furnace at atmospheric pressure and temperature up to $1223 \mathrm{~K}$ ( $950{ }^{\circ} \mathrm{C}$ ) over a period of half hour, and then cold $[11,14,4]$. It was then ground and pressed $[24,15,16]$ to form a pellet with a thickness of 3 $\mathrm{mm}$ and diameter of $12 \mathrm{~mm}$. Thus, the monocrystal was formed. The surface of this pellet was purified using acetone [2]. The steps for preparing the monocrystal are shown in figure (1).

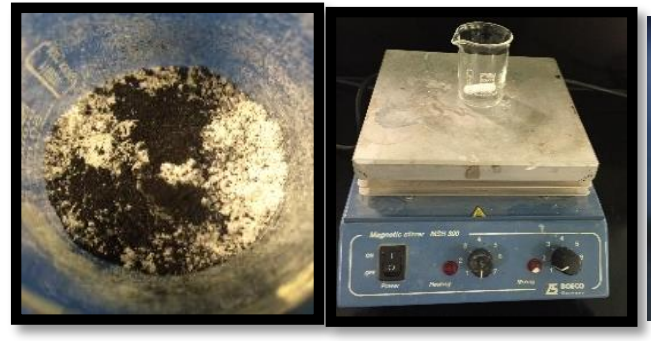

A B

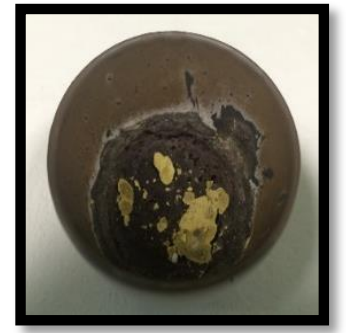

$\mathrm{D}$

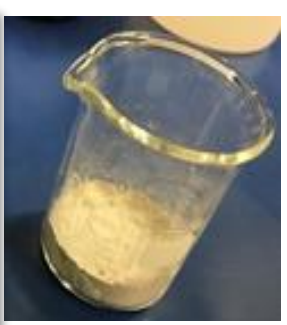

C

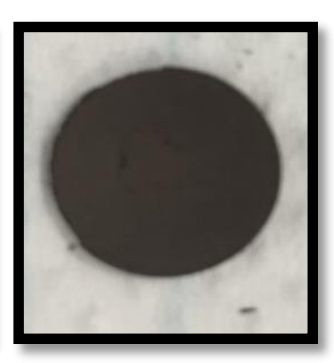

E
Figure 1. A. Powder compound mixing. B. Magnetic stirrer. C. Homogenous sample after stirring. D. Sample after annealing. E. Mn:SrTiO 3 monocrystal with thickness of $3 \mathrm{~mm}$ and diameter of $12 \mathrm{~mm}$.

\subsection{Study Methods}

XRD was used to study the structure of this monocrystal by a low-resolution device (X-ray apparatus 55481) with a molybdenum anode, maximum current intensity $\mathrm{I}=1 \mathrm{~mA}$, and voltage $\mathrm{V}=20 \mathrm{kV}$ [17], as shown in fig (2, A). The monocrystal was polished using platinum materials, and then SEM ( JSN-7610) was used to determine its grain size, as shown in figure $(2, B)$.

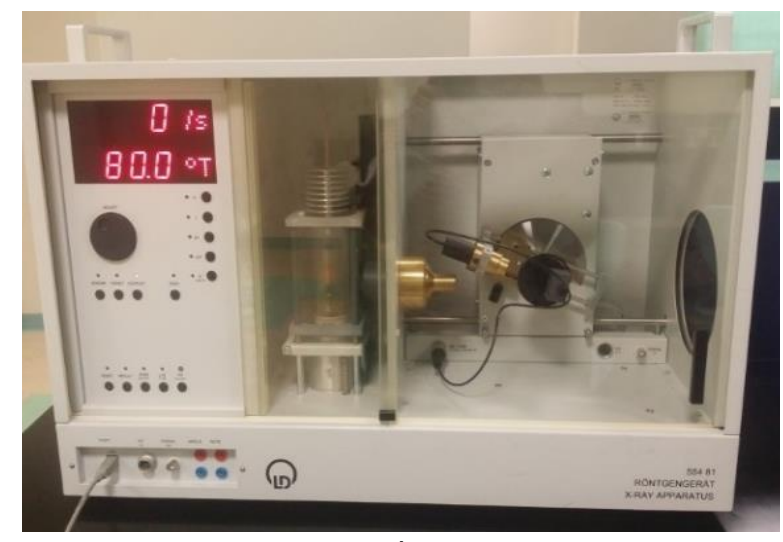

A

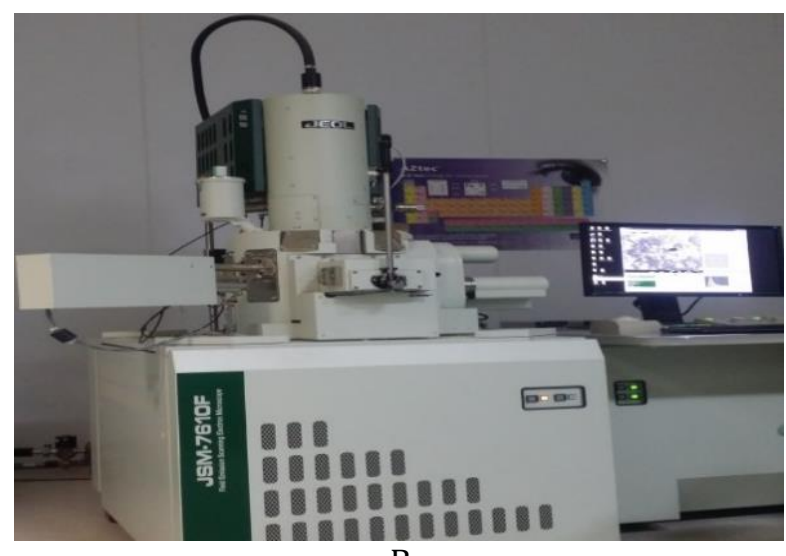

B

Figure 2. A. XRD (X-ray apparatus 55481) device. B. SEM (JSN-7610) device.

\section{Results and Discussions}

\subsection{Structural Study using XRD}

The best spectrum of this monocrystal was obtained in several steps. In the first step, the voltage was varied ( $\mathrm{V}=10,15,20$, and $25 \mathrm{kV}$ ), while the current intensity was fixed at I = $1 \mathrm{~mA}$, as shown in figure (2). From this figure, it is clear that at a high voltage, the deformation in the shape of the spectral lines is duplicated in some parts of the spectra more than in the others. This indicates a large electron emission from the cathode to the anode, which made it difficult to investigate the number of reflection planes. At a low voltage, it should be noted that the spectrum has a small number of lines. In other words, the number of reflection planes in the crystal decreased because there were several electrons in the cathode that could not gain enough energy to arrive at the anode. For this reason, the $\mathrm{X}$-rays coming from the source were very weak, which made it difficult to see the full crystal [17]. As seen in figure (3), the best spectrum of this monocrystal was clearly found at a voltage $\mathrm{V}=20 \mathrm{kV}$, and the number of 
reflection planes (lines) agrees with the other results [9]. The sample was grown in the direction of the [100] plane, based on a calculation for the axial axis.

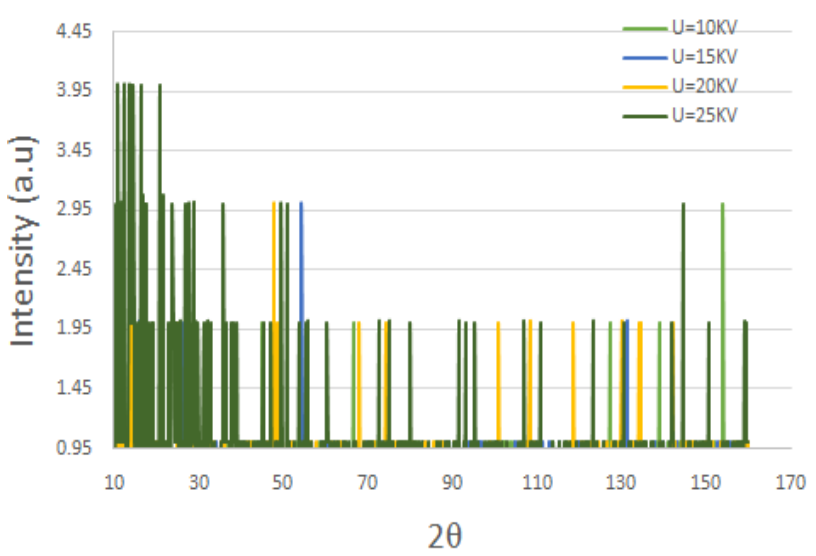

Figure 3. Spectrum lines of $\mathrm{Mn}: \mathrm{SrTiO}_{3}$ with variable voltage.

The second step involved varying the current intensity ( $\mathrm{I}=0.5,0.7,0.75$, and $1 \mathrm{~mA}$ ), while fixing the voltage $\mathrm{V}=20 \mathrm{kV}$, as shown in figure (4). At a low current intensity, the number of reflection planes decreased because the number of electrons emitted from the cathode and arriving at the anode was low, which prevented all of the planes in the crystal from being seen. The best spectrum lines found in this step were at $\mathrm{I}=1$ $\mathrm{mA}$, which agreed with the number of lines reported in the literature $[15,18]$.

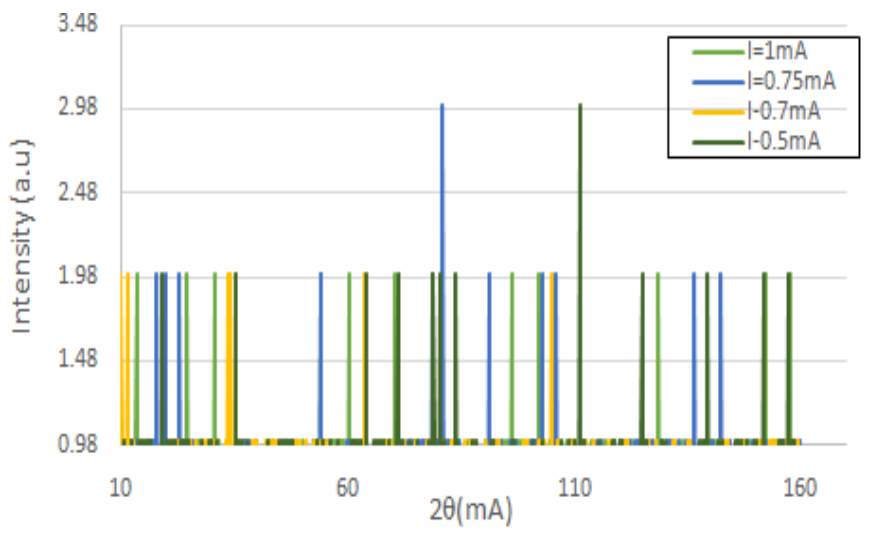

Figure 4. Spectrum lines of $\mathrm{Mn}: \mathrm{SrTiO}_{3}$ with variable current intensity.

Finally, the best spectrum was found for $\mathrm{Mn}: \mathrm{SrTiO}_{3}$ at a current intensity $\mathrm{I}=1 \mathrm{~mA}$, voltage $\mathrm{V}=20 \mathrm{kV}$, and $\mathrm{T}=300 \mathrm{~K}\left(27^{\circ} \mathrm{C}\right)$, as shown in figure (5). It consisted of eleven reflection planes, which agreed with the results of other studies $[9,18]$.

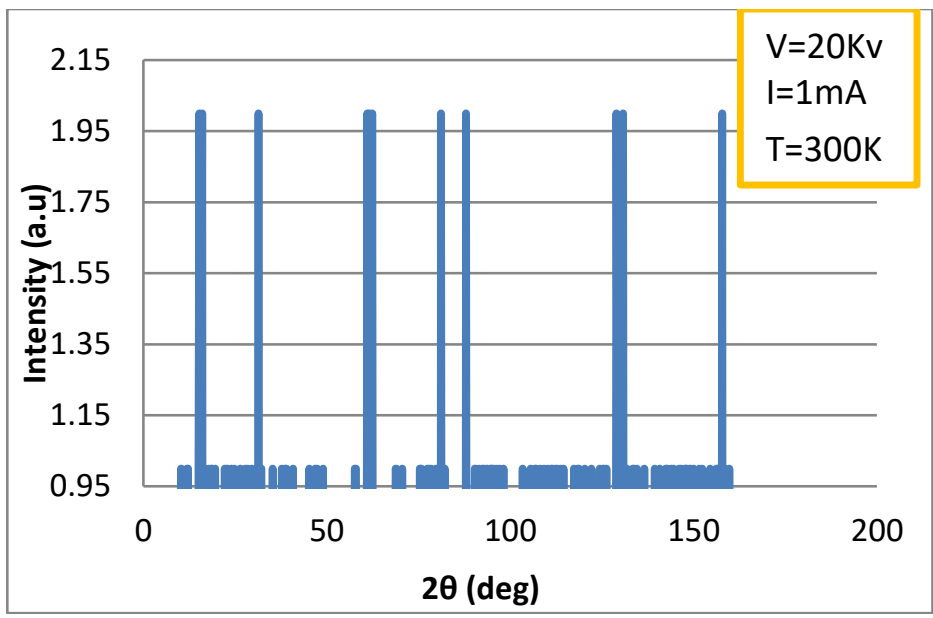

Figure 5. Best spectrum lines of $\mathrm{Mn}: \mathrm{SrTiO}_{3}$ crystal.

The X-ray wavelength of the best spectrum was calculated using equ. (1).

$$
\begin{gathered}
\lambda=\frac{h c}{e V} \\
\lambda=\frac{1.24 \times 10^{-6}}{V} \\
\lambda=0.62 \dot{A}
\end{gathered}
$$

Where $\lambda$ is wavelength, $\mathrm{h}$ is plank constant, e is electron charge and $\mathrm{V}$ is voltage. The lattice parameter (a), which was approximately equal to the distance (d) between the planes in the crystal, was calculated from the slope in figure (6) and Bragg's law (equ. (4)), as shown in the following equations [19]:

$$
\begin{gathered}
n \lambda=2 d \sin \theta \\
a=\frac{\text { slope }}{2} \\
a=3.269 \dot{A} \pm(0.008 \%)
\end{gathered}
$$

Where $\mathrm{n}$ the number of reflection planes, $\mathrm{d}$ is the distance between the reflection planes and $a$ is the lattice parameter. The lattice constant value obtained from the experimental data was nearly the same as that found by others $[8,20,9]$. The reason for the very small differences between our lattice parameter result and that of others was the ratio of Mn doped in the STO, which was very high (heavily doped) in this study 
increased, the value of the lattice parameter decreased and vice versa [20].

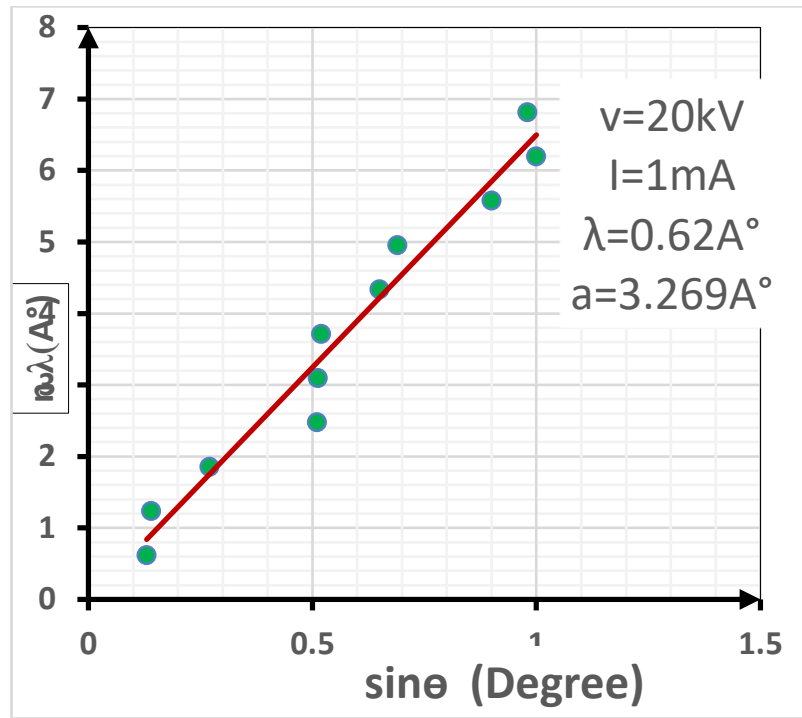

Figure 6. Relation between wavelength of reflection order $(\mathrm{n} \lambda)$ and diffraction angle $(\theta)$.

The crystal structure factor was calculated from the atomic scattering factor (f) and spectrum intensity (R), as shown in equation (7) and figure (7) [19].

$$
R_{h, k, l}=\left|f_{h, k, l}^{2}\right|
$$

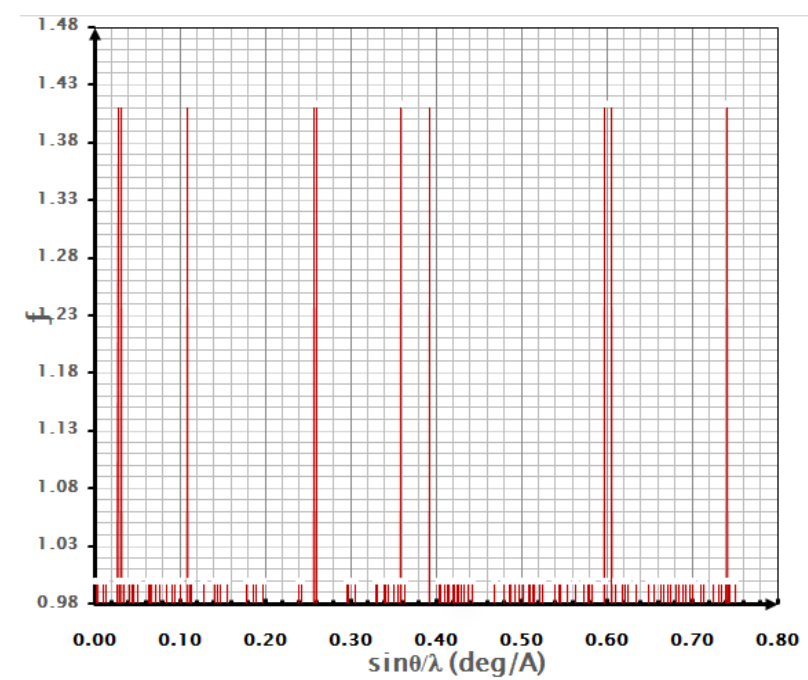

Figure 7. Relation between atomic scattering factor and $(\sin \theta / \lambda)$.

In equation (7) $\mathrm{R}$ is peak intensity, $\mathrm{h}, \mathrm{k}$, and $\mathrm{L}$ are the Miller indices in the $\mathrm{x}, \mathrm{y}$, and $\mathrm{z}$ directions, respectively. The atomic scattering factor $(F)$ is constant in figure (6). Thus, the crystal structure factor (S) can be calculated using equ. (8) and figure (6). The crystal structure had a value equal to zero, as shown in equ. (10) [21].

$$
\begin{gathered}
S=\sum_{n} f_{n} e^{2 \pi(h u+k v+l w)} \\
S=\frac{\left(f_{1}-f_{2}\right)}{\left|f_{1}-f_{2}\right|}+\Delta f \\
S=0
\end{gathered}
$$

Here, $\mathrm{n}$ is the number of atoms in the molecule; $\mathrm{u}$, $\mathrm{v}$, and $\mathrm{w}$ are the cut parts along the $\mathrm{x}, \mathrm{y}$, and $\mathrm{z}$ axes, respectively; and e is an exponential function. The structural factor was zero. Thus, this crystal was cubic, of type $(P m \overline{3} m)$, as shown in figure $(8)[21,22]$. This result agreed with those of others $[2,21,22]$, where $\mathrm{Mn}^{2+}$ substituted for $\mathrm{Sr}^{2+}$ and $\mathrm{Mn}^{4+}$ substituted for $\mathrm{Ti}^{4+}[23,1$, 10, 24].

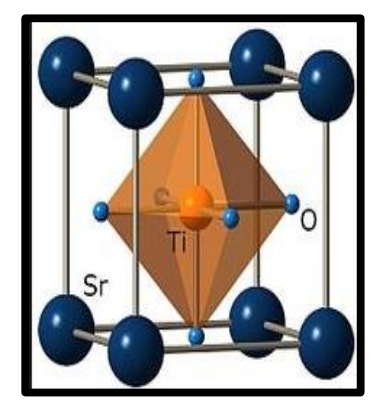

Figure 8. Structure of $\mathrm{Mn}: \mathrm{SrTiO}_{3}$ crystal $(P m \overline{3} m)$ [25].

\subsection{Calculating Grain Size using SEM Technique}

There are several techniques for elemental analyses at a specific location using SEM and transmission electron microscopy (TEM). SEM is used to analyze the surface and obtain information about the particle size, and TEM is used to determine the internal structure of a solid [26].

The grain size of this monocrystal was calculated using SEM, as shown in figure (9), with a magnification of 5,000 times. The grain size of the crystal was in the range of 1.33-1.39 $\mu \mathrm{m}$ when magnified 5,000 times, but it was in the range of 84.9-91.4 $\mathrm{nm}$ when magnified 100,000 times. The grain size changed with the level of magnification [27]. This result agreed with those of others [7]. The grain size of the crystal depended on the doping ratio of the crystal. If the doping ratio of the crystal increased, the grain size decreased [20,9]. Table (1) provides a brief summary of the results. 


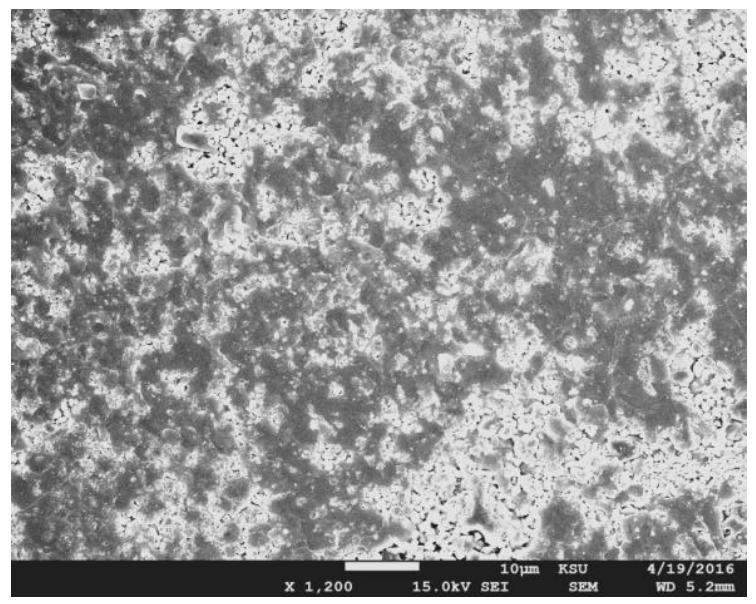

A
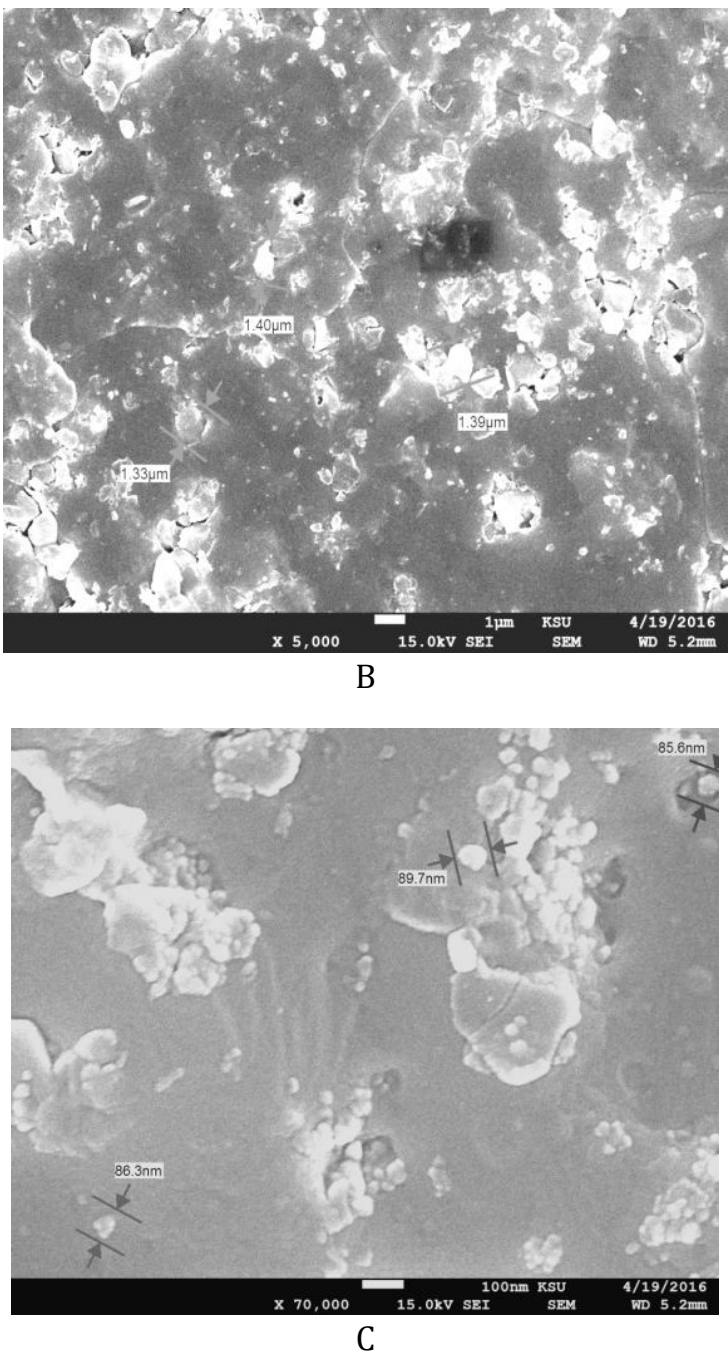

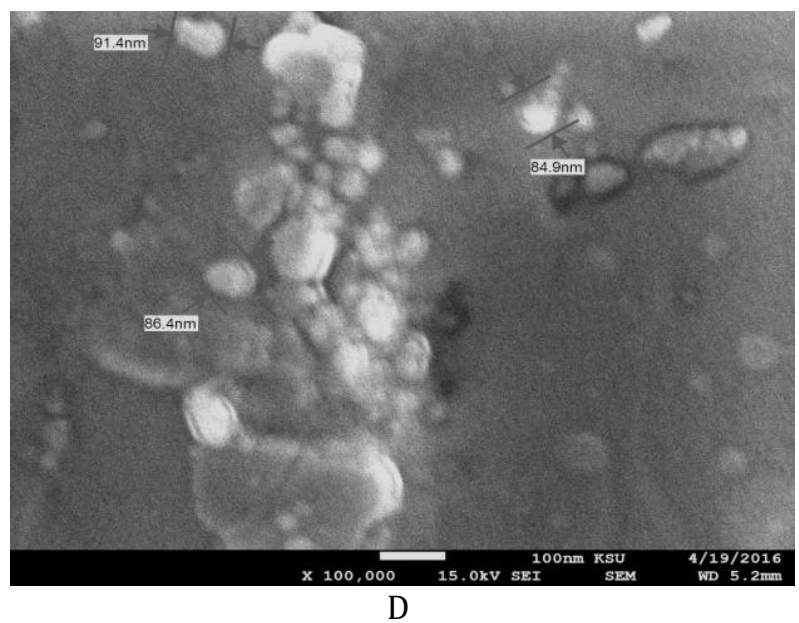

Figure 9. $\mathrm{Mn}: \mathrm{SrTiO}_{3}$ crystal at different magnifications $(1,500-100,000)$, showing differences in grain size $(1.33 \mu \mathrm{m}$ to $91.4 \mathrm{~nm})$.

Table 1 . Brief summary of results.

\begin{tabular}{|l|l|}
\hline Wavelength & $\lambda=0.62 \AA$ \\
\hline Mn ratio in crystal & $60 \%$ mole percent \\
\hline Sample size & $\begin{array}{l}\text { thickness } 3 \mathrm{~mm} \\
\text { diameter } 12 \mathrm{~mm}\end{array}$ \\
\hline Annealing temperature & $1223 \mathrm{~K}=950^{\circ} \mathrm{C}$ \\
\hline Crystal temperature & $300 \mathrm{~K}=27^{\circ} \mathrm{C}$ \\
\hline Lattice parameter $(\mathrm{a})$ & $\mathrm{a}=3.269 \AA$ \\
\hline Crystal structure type & $(P \mathrm{~m} \overline{3} \mathrm{~m})$ cubic \\
\hline Crystal grain size & $1.33 \mu \mathrm{m}$ to $91.4 \mathrm{~nm}$ \\
\hline
\end{tabular}

\section{Conclusion}

This was the first structural study of $\mathrm{Mn}: \mathrm{SrTiO}_{3}$ with $60 \mathrm{~mol} \%$ of $\mathrm{Mn}$ doped in the STO. It was investigated using a low-resolution XRD device, and the results obtained were the same as those of a neutron diffraction study of STO doped with Mn. The structure of this crystal was a cubic type $(P m \overline{3} m)$ in the direction of the [100] plane, and the lattice constant was $3.269 \AA$. The grain size was in the range $1.39 \mu \mathrm{m}-91.4 \mathrm{~nm}$ when magnified 5,000 to 100,000 times.

\section{Acknowledgment}

The authors are very grateful for the help provided by the Research Center at King Saud University in calculating the crystal grain size using SEM. We would also like to thank the physics laboratory of solar energy and some colleagues (Aml, Hana, and Hajer) in the physics and chemistry departments, who assisted us in obtaining additional research facilities. 


\section{References}

[1] F. M. F. De Groot, M. Grioni and et. al., "Strontium titanate," in Properties of strontium titanate, pp. 1-21, 2016.

[2] D. Choudhury, "Site-specific doping, tunable dielectric properties, and intrinsic paramagnetism in Mn-doped SrTiO3," Pramana J. Phys., vol. 84, no. 6, pp. 987-997, 2015.

[3] M. D. Biegalski, D. D. Fong and e. al., "Critical thickness of high structural quality SrTi03 films grown on orthorhombic (101) DySc03," Journal of Applied Physics, vol. 104, pp. 114109, 2008.

[4] T. Fix, F. Schoofs and et. al., "Influence of doping at the nanoscale at LaALO3:SrTiO3 interfaces," Appl. Phys. lett., pp. 072110-1072110-3, 2010.

[5] L. P. Gor'kov, "Phonon mechanism in the most dilute superconductor: n type SrTiO3," in PNAS, Tallahassee, vol. 28, pp. 4646-4651, 2016.

[6] M. C. Gallardo, A. I. Becerro and et. al., "Cubictetragonal phase transition in $\mathrm{Ca}_{0.04} \mathrm{Sr}_{0.96} \mathrm{TiO}_{3}$ : A combined specific heat and neutron diffraction study," J. Phy. Condens. Matter, vol. 15, no. 2, pp. 91-100, 2002.

[7] R. M. Mohamed, M. M. Rashad and et. al., "Structure and magnetic properties of nanocrystalline cobalt ferrite powders synthesized using organic acid precursor method," J. Magn. and Magn. Mat., vol. 322, no. 14, pp. 2058-2064, 2010.

[8] C. B. Azzoni, M. C. Mozzati and et. al., "Magnetic investigation of Mn ions in La,Mn:SrTiO," Solid State Communications, vol. 116, no. 6, pp. 303308, 2000.

[9] D. Choudhury, R. M. Goodbole and et. al., "Top polarization, forward-backward asymmetry, and new physics," Phys. Rev D., vol. 84, pp. 125-124, 2011.

[10] D. Choudhury, S. Mukherjee and et. al., "Tuning of dielectric properties and magnetism of $\mathrm{SrTiO} 3$ by site-specific doping of Mn," A. B. Phys., vol. 53, pp. 5061-5069, 2005.

[11] J. Choi, C. B. Eom and et. al., "Growth mode transition from layer by layer to step flow during the growth of heteroepitaxial $\mathrm{SrRuO} 3$ on (001) SrTiO3," Appl. Phys. Lett., vol. 79, no. 10, pp. 1449-1447, 2001.

[12] M. Kareev, S. Prosandeev and et. al., "Atomic control and characterization of surface defect states of $\mathrm{TiO} 2$ terminated $\mathrm{SrTiO} 3$ single crystals," Appl. Phys. Lett., vol. 93, pp. 202-228, 2008.
[13] J. Zhang, D. Doutt and et. al, "Depth-resolved subsurface defects in chemically etched SrTiO3," Appl. Phys. Lett., vol. 94, pp. 092904-092908, 2009.

[14] A. Abadpour, "A sequential Bayesian alternative to the classical parallel fuzzy clustering model," Information Sciences, vol. 318, pp. 28-47, 2015.

[15] A. M. Dehkordi, S. Bhattacharya and et. al., "New insights on the synthesis and electronic transport in bulk polycrystalline $\mathrm{Pr}$-doped SrTiO3- $\delta$," Journal of Applied Physics, vol. 117, pp. 055102, 2015.

[16] M. S. J. Mashall and M. R. Castell, "Shape transitions of epitaxial islands during strained layer growth: Anatase TiO3(001) on SrTiO3(001)," Physical Review Letters, vol. 102, pp. 146-102, 2009.

[17] L. D. Gmbtt, "Instruction sheet 554 82," Hurth, 2001.

[18] A. El-Sayed, "Influence of zinc content on some properties of Ni-Zn ferrites," Ceramics

International, vol. 28, 2002, pp. 363-367.

[19] H. Kooijman, "Interpretation of crystal struncture determinations," Bijvoet Center for Biomolecular Research, CH Utrecht, 2005.

[20] C. Azzoni, M. C. Mozzati and et. al, "Magnetic evidence of different environments of manganese ions in Mn-substituted strontium titanate," Solid State Communications, vol. 114, no. 12, pp. 617622, 2000.

[21] M. Roessle, "Basic of x-ray scattering," EMBO course 2012, 2009.

[22] M. Huijben, A. Brinkman and et. al, "Structureproperty relation of SrTiO3-LaAlO3 interfaces," Advanced Materials, vol. 21, no. 17, pp. 16651677, 2009.

[23] F. R. Silly, M. Castell and et.al., "Bimodal growth of Au on SrTiO3_001," Physical Review Letters, vol. 96, no. 8, pp. 1-4, 2006.

[24] A. Tkach, P. Vilarinho and e.al. "Structuremicrostructure-dielectric tunability relationship in Mn-doped strontium titanate ceramics," Acta Materialia, vol. 53, no. 19, pp. 5061-5069, 2005.

[25] Postnova. (2016) Centrifugal field-flow fractionation principle [Online]. Available: http://www.postnova.com/centrifugalfff.html?gclid=CMbNkZ6Tg88CFYUy0wodgoAM $\mathrm{Eg}$

[26] T. Leisegang, H. Stöcker, and et. al, "Switching Ti Valence in SrTiO3 by a dc Electric Field," Phys. Rev. Lett. vol. 102, pp. 087601, 2009. 
[27] Deft Solid Solutions. (2016). Scanning \& transmission electron microscopy. [Online].

Available: https://www.solids-

solutions.com/rd/particle-sizing-and-particle-size-

analysis/scanning-transmission-electron-

microscopy/ 